Interfaces

\title{
Les gestes de la musique ancienne dans les textes et l'iconographie
}

\section{Cyril Lacheze et Marion Weckerle}

\section{(2) OpenEdition}

1 Journals

Édition électronique

URL : http://journals.openedition.org/interfaces/490

DOI : 10.4000/interfaces.490

ISSN : 2647-6754

Éditeur :

Université de Bourgogne, Université de Paris, College of the Holy Cross

\section{Édition imprimée}

Date de publication : 1 juillet 2018

Pagination : 91-114

ISSN : 1164-6225

\section{Référence électronique}

Cyril Lacheze et Marion Weckerle, « Les gestes de la musique ancienne dans les textes et

l'iconographie », Interfaces [En ligne], 39 | 2018, mis en ligne le 01 juillet 2018, consulté le 05 janvier 2021. URL : http://journals.openedition.org/interfaces/490 ; DOI : https://doi.org/10.4000/interfaces. 490

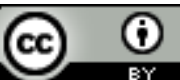

Les contenus de la revue Interfaces sont mis à disposition selon les termes de la Licence Creative Commons Attribution 4.0 International. 


\title{
LES GESTES DE LA MUSIQUE ANCIENNE DANS LES TEXTES ET L'ICONOGRAPHIE
}

\author{
Cyril Lacheze \& Marion Weckerle \\ IHMC, Université Paris 1 Panthéon-Sorbonne
}

L'évolution des techniques de jeu instrumentales dans la musique européenne a conduit à des modifications radicales des gestes mis en œuvre par les interprètes au fil du temps, avec un oubli progressif des techniques anciennes et des implications sonores que celles-ci entraînaient. Le mouvement dit « historiquement informé » de redécouverte de la pratique originelle de cette musique ancienne, entamé il y a presque un demi-siècle, a donc impliqué un travail de reconstitution de ces gestes appuyé sur une analyse précise des sources disponibles, souvent éparses (Boyden). Si, dans un premier temps, ces recherches se sont contentées de remettre au point une technique ancienne " générique ", une tendance actuelle vise à accentuer encore le degré de précision pour identifier les gestes associés à chaque contexte chrono-géo-sociologique de pratique musicale (Moens-Haenen). De plus, si cette approche de la musique ancienne s'est longtemps cantonnée aux mouvements Renaissance, baroque et classique (soit l'équivalent musical de l'époque moderne), elle s'approprie de plus en plus également le Romantisme du XIX ${ }^{\text {ème }}$ siècle et la période moderne du début du XX $^{\text {ème }}$ siècle, puisque les instruments et la technique de jeu employés actuellement se sont souvent fixés autour du tiers, voire du milieu du $\mathrm{XX}^{\mathrm{ème}}$ siècle.

Le questionnement est ainsi à double sens dans cette étude : d'une part, il s'agit de chercher à comprendre les gestes disparus dans une démarche analytique historienne afin de cerner leurs modalités d'existence et d'évolution, et de définir ainsi une dynamique concernant leur existence en tant que fait historique, ce à partir des sources de diverses natures qui nous sont connues. Toutefois, ce résultat sert lui-même de point de départ à la démarche de restitution propre à la musique ancienne, discipline artistique bien vivante dans laquelle ces conclusions sont mises en application d'une manière très concrète pour jouer la musique dans une démarche de recherche de " plausibilité », à défaut d' " authenticité » (idéalement et virtuellement recherchée mais par définition inatteignable). Le résultat obtenu, pouvant être considéré comme une expérimentation des techniques identifiées via l'analyse des sources, peut d'ailleurs lui-même être réintégré dans l'analyse de celles-ci, formant ainsi un continuum entre ces deux démarches. 
Nous nous intéresserons ici aux deux aspects de la recherche, étude du geste ancien et mise en pratique de celui-ci dans le jeu musical actuel, ce à travers les exemples de deux instruments. En premier lieu, jusqu'au XVIII ${ }^{\text {ème }}$ siècle, nous nous appuierons sur le cas du violon, instrument qui s'est développé principalement à cette période. À la fois le matériel et sa technique de jeu sont apparus dans le premier tiers du XVI ${ }^{\text {ème }}$ siècle, et se sont fixés sur des formes proches de leur état actuel à quelques détails près au tournant des XVIII ${ }^{\text {ème }}$ et XIX ${ }^{\text {ème }}$ siècles. Pendant l'époque baroque, il était l'instrument phare dans la plupart des catégories sociales, aussi bien populaires qu'à la cour, bien que les milieux bourgeois se tournaient plutôt vers la viole de gambe. Il a ainsi généré l'une des littératures et des iconographies les plus abondantes parmi les instruments de cette époque. Pour les périodes plus récentes, nous prendrons l'exemple de la clarinette : apparue au tournant des XVII ${ }^{\text {ème }}$ et XVIII ${ }^{\text {ème }}$ siècles, celle-ci n'a toutefois connu un développement marqué qu'à partir de la fin du XVIII ${ }^{\text {me }}$ siècle, sa facture, et donc sa technique de jeu, évoluant lourdement par l'ajout progressif de clés tout au long du $\mathrm{XIX}^{\text {ème }}$ siècle, pour ne se fixer qu'au début du $\mathrm{XX}^{\mathrm{ème}}$ siècle. Elle est en conséquence abondamment documentée par des méthodes et traités publiés par des professeurs renommés (Beer 1836, Lefèvre 1802, Van Der Hagen 1785) tout au long du XIX ${ }^{\text {ème }}$ siècle, accompagnant la pratique en conservatoire qui se développait alors.

\section{Les gestes et les sources}

Le type de source le plus habituellement employé pour l'étude des gestes du jeu des instruments est constitué par des textes généralement imprimés et pouvant correspondre soit à des traités théoriques, principalement pour le XVI ${ }^{\text {ème }}$ et le début du XVII ${ }^{\text {ème }}$ siècles, où ils participaient de la logique de la réduction en art (Dubourg-Glatigny et Vérin), et plus souvent à des méthodes, particulièrement courantes à partir de la fin du XVII ${ }^{\text {ème }}$ siècle. Celles-ci suivaient généralement toujours le même schéma. Elles commencent par quelques pages introductives balayant souvent toute la progression potentielle de l'apprenant, débutant par l'explication de l'écriture de la musique et présentant l'instrument, pour finir par des techniques plus ou moins avancées. Ces introductions sont toujours d'actualité dans les méthodes actuelles d'apprentissage mais sont très rarement mises à contribution : beaucoup trop succinctes pour être réellement utiles à l'apprentissage, elles servent au mieux d'aide-mémoire, le professeur se chargeant d'indiquer à l'apprenant ce qu'il doit savoir. Cette introduction, éventuellement agrémentée de quelques schémas, constitue cependant la principale source d'information sur le geste pour le chercheur, sachant que les indications techniques correspondent rarement à plus d'un paragraphe, parfois seulement à quelques mots, voire sont souvent totalement absentes. Le reste de ces méthodes était intégralement composé de partitions, correspondant à un cahier d'exercices (gammes, études et 
petits morceaux) de difficulté progressive que le professeur faisait travailler à l'élève, parfois en jouant avec lui, et qui ne comportaient généralement quasiment aucune indication de type technique imprimée. À notre connaissance, aucun exemplaire annoté à la main par un professeur n'est parvenu jusqu'à nous.

L'autre source importante, au moins pour certains instruments dont le violon, est l'iconographie : outre un certain nombre de gravures représentant notamment les fêtes de cours françaises, le violon apparaît dans nombre de tableaux donnant à observer des scènes de genre, et dans les mains d'anges sur d'autres représentations à sujets religieux. Celles-ci posent toutefois à l'évidence la question de leur plausibilité. Certaines images ne sont clairement pas réalistes, parce que le geste montré est physiologiquement impossible, ou encore à cause d'un détail manquant sur l'instrument qui remet en doute la précision de l'ensemble. Toutefois, dans les cas où la scène montrée est effectivement réaliste, il est impossible d'assurer que celle-ci renvoie bien à la technique employée à l'époque. Le peintre aurait très bien pu représenter un instrumentiste de manière réaliste mais en suivant uniquement son imagination, ou encore suivre un modèle qui, pour une raison ou une autre, n'aurait pas suivi la technique habituelle de son époque. Ainsi, seul le poids statistique fourni par la répétition d'un même geste dans de nombreux tableaux d'un contexte chrono-géo-sociologique donné permet d'avancer l'hypothèse que la technique figurée a bien pu être utilisée alors. En conséquence, il convient de travailler sur un nombre aussi élevé que possible de représentations, les bases de données en ligne permettant à l'heure actuelle d'en regrouper quelques centaines, mais qui sont souvent concentrées sur certaines époques et qui laissent des pans entiers de la plage chronologique considérée dans l'ombre, tout comme les traités d'ailleurs.

On constate en effet de fortes disparités dans la répartition des deux types de sources, qui se corrèlent d'ailleurs rarement et sont pour autant loin de se compléter parfaitement. En ce qui concerne le violon à l'époque moderne, on connaît une trentaine de traités et méthodes comportant des informations, même minimes, sur les gestes techniques qui conditionnaient son jeu (Figure 1); à titre d'exemple quelque peu extrême, on citera l'indication du traité de Trichet vers 1640 dans son intégralité : "on le [le violon] tient appuié contre l'espaule gauche » (Trichet 109). Quatre ouvrages seulement, pour la plupart français, apparaissent entre le milieu du XVI ${ }^{\text {ème }}$ et le milieu du XVII ${ }^{\text {ème }}$ siècles. Le dernier quart du XVII ${ }^{\text {ème }}$ siècle est bien documenté en Allemagne, région où l'on relève encore régulièrement des parutions tout au long du siècle suivant. La France est l'autre pays bien documenté du XVIII ${ }^{\text {me }}$ siècle, avec une dizaine de titres. S'y ajoute plus ou moins l'Angleterre, laquelle a vu régulièrement pendant un siècle à partir de 1664 l'édition de fascicules destinés à un public bourgeois, qui ne comportent que de très rares informations et se recopient sans forcément réaliser de mise à jour ; la seule méthode à proprement parler en Angleterre est celle de Geminiani, en 1751. Enfin, les autres régions européennes, et notamment l'Italie, pourtant motrice dans le développement de la technique violonis- 
tique à l'époque, sont presque totalement absentes. En ce qui concerne les représentations picturales (Figure 2) ${ }^{1}$, l'Italie se place en revanche nettement en tête, en particulier au XVI ${ }^{\text {ème }}$ siècle, mais avec des sujets principalement religieux qui sont généralement les plus problématiques en termes de plausibilité de la représentation. L'Allemagne de la Renaissance est également assez documentée, mais surtout la Hollande du XVII ${ }^{\text {ème }}$ siècle, avec les innombrables scènes de tavernes comme d'intérieurs aisés dépeints par les maîtres du baroque flamand. Le XVIII ${ }^{\text {ème }}$ siècle fait cette fois figure de parent pauvre, avec uniquement une certaine production française, les œuvres anglaises étant pour beaucoup des caricatures quasiment dépourvues de pouvoir informatif.

\begin{tabular}{|c|c|c|c|c}
\hline Dates & Allemagne & Angleterre & France & Espagne \\
\hline 1556 & & & Jambe de Fer & \\
\hline 1589 & & & Arbeau & \\
\hline 1618 & Praetorius & & & \\
\hline v. 1640 & & & Trichet & \\
\hline 1664 & & Playford & & \\
\hline 1677 & Prinner & & & \\
\hline 1687 & Speer & & & \\
\hline 1688 & Falck & & & \\
\hline 1693 & & Lenton & & \\
\hline 1695 & Merck & & & \\
\hline 1698 & Muffat & & & \\
\hline 1711 & & & Monté clair & \\
\hline v.1711 & & & De Brossard & \\
\hline 1722 & & [Pearson] & & \\
\hline 1732 & Majer & & & \\
\hline v. 1735 & & Crome & & \\
\hline 1738 & & & Corrette & \\
\hline 1751 & & Geminiani & Diderot & \\
\hline 1756 & Mozart & [Sadler $]$ & & De Herrando \\
\hline 1761 & & & L'Abbé Le Fils & \\
\hline 1763 & Kürzinger & & & \\
\hline 1770 & Mozart & & & \\
\hline 1772 & & & Labadens & \\
\hline 1774 & Löhlein & & Tarade & \\
\hline 1780 & & & De Laborde & \\
\hline 1782 & & & Corrette & \\
\hline & & & & \\
\hline
\end{tabular}

Figure 1 : Traités utilisés présentant des informations sur la tenue du violon (détail des œuvres dans les Ouvrages Cités).

Ceci représente des centaines de sources que nous ne pouvons pas lister ici, mais que l'on trouvera aisément en ligne (Lacheze). 


\begin{tabular}{|c|c|c|c|c|c|c|c|c|c|c|}
\hline \multirow{2}{*}{ Période } & \multirow{2}{*}{$\begin{array}{l}\text { Type de } \\
\text { sujet }\end{array}$} & \multicolumn{9}{|c|}{ Pays d'origine de l'artiste } \\
\hline & & Italie & Espagne & France & Suisse & Allemagne & Bohême & Hollande & Belgique & Angleterre \\
\hline \multirow{4}{*}{ Moyen Âge } & Populaire & & & & & & & & & \\
\hline & Bourgeois & & & & & 1 & & & & \\
\hline & Cours & & & & & & & & & \\
\hline & Religieux & 56 & 1 & 2 & 3 & 12 & & 3 & & 1 \\
\hline & & & & & & & & & & \\
\hline \multirow{4}{*}{ Renaissance } & Populaire & & & & & & & 1 & & \\
\hline & Bourgeois & 3 & & & & 2 & & & & \\
\hline & Cours & 1 & & & & 3 & & & & \\
\hline & Religieux & 36 & & & 1 & 12 & & 9 & & 1 \\
\hline & & & & & & & & & & \\
\hline \multirow{4}{*}{$\begin{array}{l}\text { Baroque } \\
\text { ancien }\end{array}$} & Populaire & 1 & & & & & & 15 & & \\
\hline & Bourgeois & 6 & 1 & 2 & & & & 20 & & \\
\hline & Cours & & & & & & & 1 & & 3 \\
\hline & Religieux & 14 & 2 & 3 & & 1 & & 5 & 3 & 1 \\
\hline & & & & & & & & & & \\
\hline \multirow{4}{*}{$\begin{array}{l}\text { Baroque } \\
\text { moyen }\end{array}$} & Populaire & & & 1 & & & 1 & 23 & 1 & \\
\hline & Bourgeois & & & 1 & & & & 17 & & \\
\hline & Cours & 1 & & 1 & & & & 1 & & \\
\hline & Religieux & 2 & 2 & 1 & & 1 & & 1 & 1 & \\
\hline & & & & & & & & & & \\
\hline \multirow{4}{*}{$\begin{array}{c}\text { Baroque } \\
\text { récent }\end{array}$} & Populaire & 5 & & & & 1 & & & & 8 \\
\hline & Bourgeois & 1 & & 11 & & 2 & & & & 3 \\
\hline & Cours & 1 & 1 & & & & & & & \\
\hline & Religieux $x$ & 2 & & 2 & & 4 & & & & \\
\hline & & & & & & & & & & \\
\hline \multirow{4}{*}{ Classicisme } & Populaire & & & & & 1 & & & & 1 \\
\hline & Bourgeois & 1 & & 4 & & 4 & & & & \\
\hline & Cours & & & 1 & & & & & & 1 \\
\hline & Religieux & 1 & & & & & & & & \\
\hline
\end{tabular}

Figure 2 : Iconographie utilisée présentant des informations sur la tenue du violon (détail des œuvres dans Lacheze).

En s'appuyant sur ces différentes sources, il est possible de renseigner les habitudes techniques d'environ la moitié des espaces chrono-géographiques définissables (par grandes zones culturelles et périodes temporelles d'environ un demi-siècle), chacun étant souvent connu par un seul type de source, et l'autre moitié restant trop peu documentée pour pouvoir apporter des informations sûres (Figure 3). Dans tous les cas, la tenue représentée est effectivement distincte de la pratique actuelle, qui consiste à tenir le violon directement contre le cou avec le menton posé dessus, et l'archet à l'extrémité (sur la hausse), avec le pouce entre la baguette et la mèche, et tous les autres doigts posés sur la baguette. 
Jusqu'à la seconde moitié du XVII ${ }^{\text {ème }}$ siècle, le violon était tenu très bas, contre la poitrine ou l'épaule, représentation omniprésente dans la peinture flamande (Figure 4), correspondant bien à l'indication de Trichet citée ci-dessus par exemple. L'archet était quant à lui à la hausse mais avec le pouce sous la mèche (ou plutôt sous la hausse), en lien avec l'usage d'archets courts et une musique particulièrement dynamique (tenue souvent appelée « à la française » d'après une explication donnée par Corrette en 1738 (Figure 5). Les premiers exemples d'une tenue plus haute du violon, contre la clavicule voire le cou, s'observent en France et en Angleterre à la fin du XVII ${ }^{\text {ème }}$ siècle, avant de se généraliser au XVIII ${ }^{\text {ème }}$ siècle ; la tenue « italienne » de l'archet ${ }^{2}$, avec le pouce sous la baguette et non sous la mèche pour accompagner un allongement progressif des archets, apparaît à la même période. Il faut toutefois noter que Léopold Mozart, au début de la période classique dans la seconde moitié de ce siècle, trouvait toujours la tenue basse du violon plus élégante et ne recommandait la tenue haute que pour la facilité que celle-ci procure pour démancher ${ }^{3}$ grâce à la possibilité de poser le menton sur le violon (Mozart 1756 53). De plus, la tenue actuelle, avec le menton posé en permanence sur l'instrument, n'est mentionnée pour la première fois qu'en 1774, par Löhlein, et voisinait alors toujours avec les variantes plus anciennes. Ainsi, on peut observer une évolution lente et continue de ces gestes techniques, dont les formes actuelles étaient encore à peine apparues à la fin du XVIII ${ }^{\text {ème }}$ siècle.

\begin{tabular}{|c|c|c|c|c|c|c|c|c|c|c|}
\hline & \multicolumn{2}{|c|}{ Italie } & \multicolumn{2}{|c|}{ France } & \multicolumn{2}{|c|}{ Allemagne } & \multicolumn{2}{|c|}{ Hollande } & \multicolumn{2}{|c|}{ Angleterre } \\
\hline & Vl. & Ar. & V1. & Ar. & Vl. & Ar. & Vl. & Ar. & Vl. & Ar. \\
\hline Renaissance (1500-1580) & \multirow{2}{*}{ Basse } & \multirow{2}{*}{ Fr. } & & & & & & & & \\
\hline Baroque ancien (1580-1630) & & & Basse & \multirow{3}{*}{ Fr. } & & & \multirow{2}{*}{ Basse } & Fr. & & \\
\hline Baroque moyen (1630-1700) & & & Hout & & & & & Var. & Var. & \multirow{2}{*}{ It. } \\
\hline Baroque récent (1700-1750) & \multirow{2}{*}{ Haute } & \multirow{2}{*}{ Var. } & Hatue & & Haute & It. & & & Haute & \\
\hline Classicisme (1750-1800) & & & & & & & & & & \\
\hline
\end{tabular}

Vl. : Violon (tenue)

Fr. : [Tenue] « française » (à la hausse)

Ar. : Archet (tenue)

It. : [Tenue] « italienne » (avancée sur la baguette)

Var. : [Tenue] variable

Figure 3 : Tableau récapitulatif des gestes techniques déduits de l'observation des sources pour le violon, d'après Lacheze (232).

Toujours d'après la terminologie de Corrette, on se référera à la Figure 3 pour constater que les choses ne sont manifestement pas aussi tranchées dans la réalité.

3 Déplacer la main gauche le long du manche pour jouer des notes aigües. 


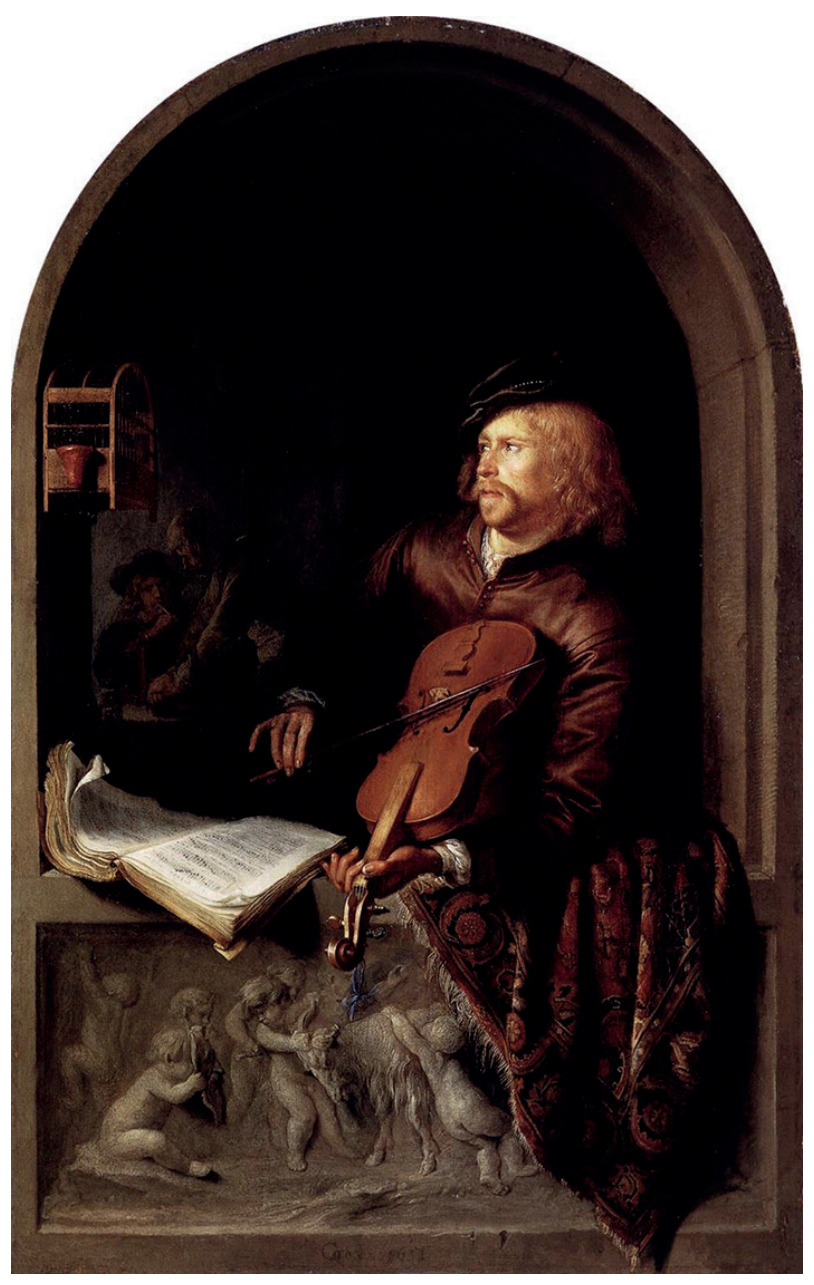

Figure 4 : Gerrit Dou. Violoniste (1653). Huile sur toile, 32 x $20 \mathrm{~cm}$. Liechtenstein Museum, Vienne. 


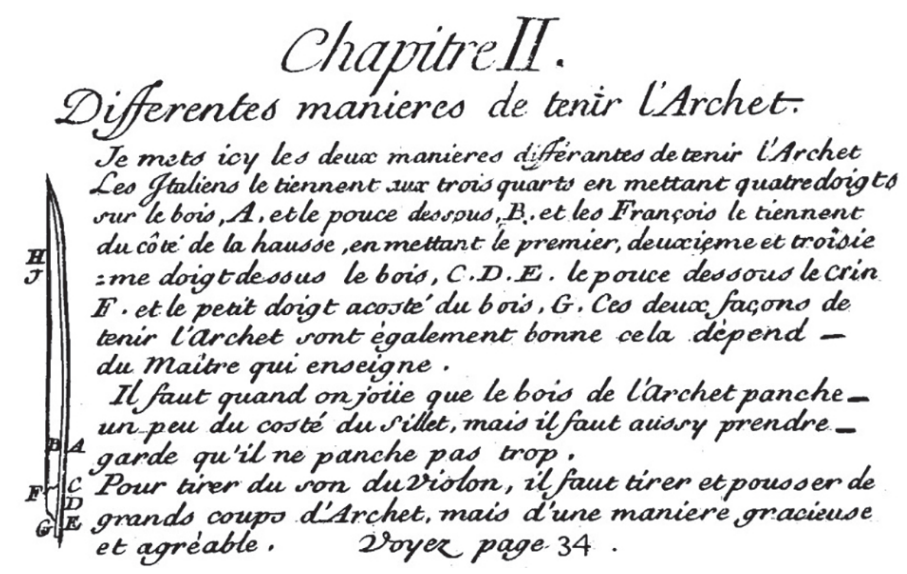

Figure 5 : Le paragraphe concernant la tenue de l'archet dans Michel Corrette, L'École d'Orphée (1738), p. 7.

En ce qui concerne la clarinette, les gestes à traquer sont, outre la posture générale du corps, les techniques d'emploi des doigts, des lèvres, des dents et de la langue. Ces détails ne sont pas identifiables sur l'iconographie représentant des clarinettes, lorsque l'instrument est seulement nommé correctement. Par exemple, le musicien dans L'homme assis près d'un arbre jouant de la clarinette, dessin de la fin du XVI ${ }^{\text {ème }}$ siècle conservé au musée du Louvre, joue en réalité de la chalemie ${ }^{4}$. Même l'instrument est rarement bien visible, à de rares exceptions près, en particulier une toile de FrançoisLéonard Dupont-Watteau (Gétreau) (Figure 6), et un tableau du bavarois Krämer de la fin du XVIII ${ }^{\text {ème }}$ siècle représentant une clarinette et son anche avec grande précision (Pearson 89). L'iconographie existante n'est donc pas réellement exploitable, et les méthodes rédigées à partir de la fin du XVIII ${ }^{\text {ème }}$ siècle constituent la source la plus pertinente. Leurs auteurs sont des clarinettistes au talent reconnu à leur époque, comme plusieurs membres du Concert Spirituel avant la Révolution française, ainsi que des professeurs de clarinette, en particulier des professeurs du Conservatoire de Paris (actuellement CNSMDP) après la Révolution, et des éditeurs de musique qui cherchaient à toucher également un public amateur.

Paul Stockher. Homme assis près d'un arbre jouant de la clarinette. Dessin à la plume. 1592. Musée du Louvre, INV 19222, Paris. 


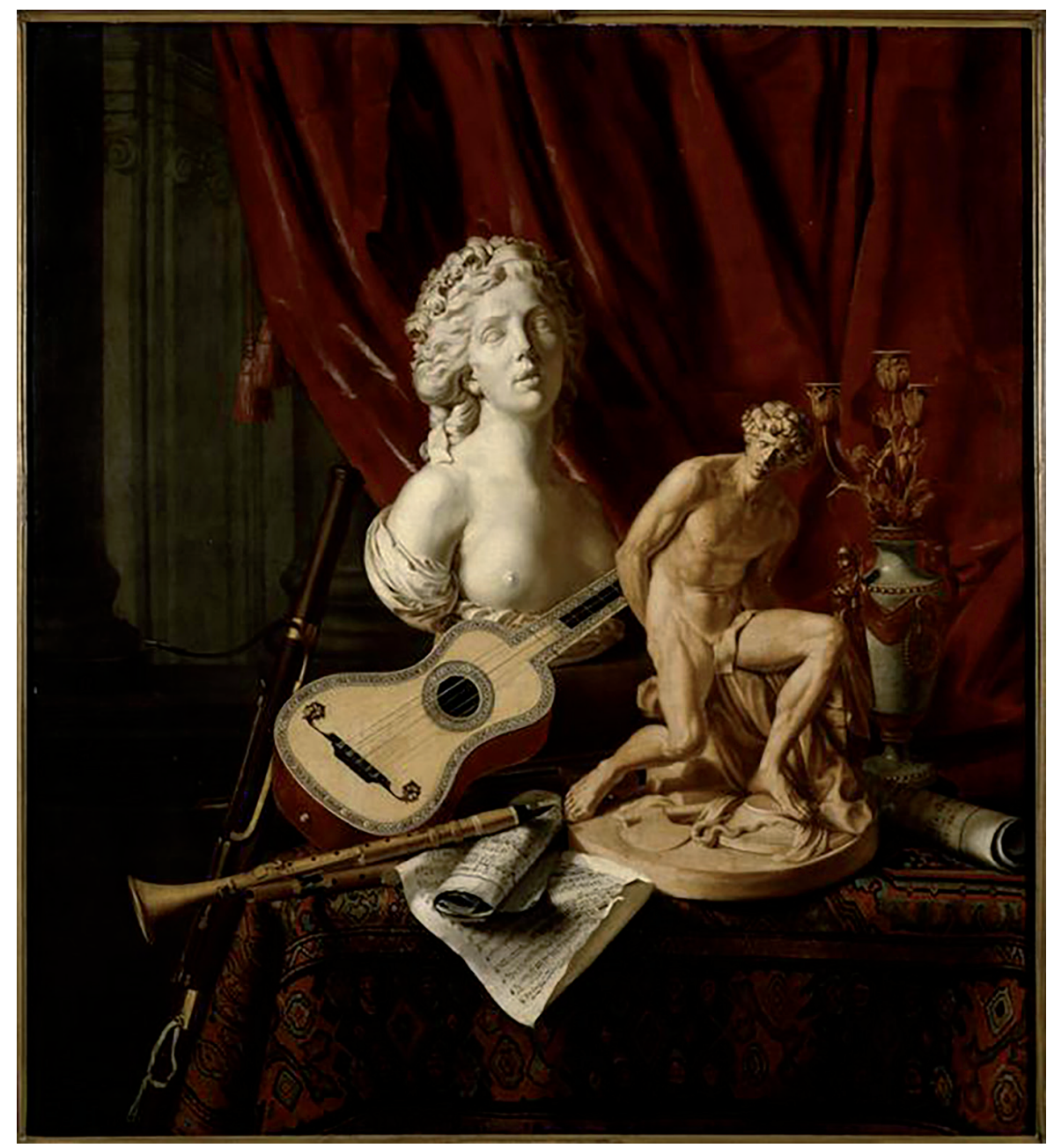

Figure 6 : François-Léonard Dupont-Watteau, Attributs des arts (1785).

Huile sur toile, 151 x $140 \mathrm{~cm}$. Palais des Beaux-Arts, Lille. Photo RMN-Grand Palais/Philipp Bernard. 


\begin{tabular}{|c|c|c|c|c|c|}
\hline Date & Allemagne & France & $\begin{array}{l}\text { Royaume- } \\
\text { Uni }\end{array}$ & Italie & États-Unis \\
\hline 1732 & Majer & & & & \\
\hline 1738 & Eisel & & & & \\
\hline 1758 & Adlung \& Bach & & & & \\
\hline 1760 & & Roeser & & & \\
\hline 1773 & & Corrette & & & \\
\hline 1775 & & Hoteterre & & & \\
\hline 1780 & & & Anonyme & & \\
\hline 1782 & & Abraham & & & \\
\hline 1785 & & Van Der Hagen & & & \\
\hline 1790 & A Ibrechts berger & Anonyme & & & \\
\hline 1796 & & $\begin{array}{l}\text { Blasius. Van } \\
\text { Der Hagen }\end{array}$ & & & \\
\hline 1800 & & Yost & & & \\
\hline 1802 & & Lefèvre & & & \\
\hline 1804 & & & & & Blake \\
\hline 1808 & & Bochsa, Démar & & & \\
\hline 1810 & Frôhlich & & & & \\
\hline 1813 & & & & Antolini & \\
\hline 1821 & Müller & & & & \\
\hline 1824 & Backo fen & & & & \\
\hline 1826 & & $\begin{array}{l}\text { Rybicki, } \\
\text { Vaillant }\end{array}$ & & & \\
\hline 1829 & & Carnaud & & & \\
\hline 1836 & & Berr & & & \\
\hline 1840 & & Gambaro & & & \\
\hline 1841 & & Nadaud & & & \\
\hline 1850 & & & & & Howe \\
\hline 1851 & & Nadaud & & & \\
\hline 1855 & & Cornette & & & \\
\hline 1865 & Baermann & & & & \\
\hline 1868 & & Kellner & & & \\
\hline 1875 & & Boscher & & & \\
\hline 1876 & & Bouillon & & & \\
\hline 1879 & & Kellner & & & \\
\hline 1881 & & & De Ville & & \\
\hline 1891 & Beer & & & & \\
\hline 1900 & Magnani & & & & \\
\hline
\end{tabular}

Figure 7 : Synthèse des méthodes de clarinette du XVIII ${ }^{\text {ème à la fin du XIX }}{ }^{\text {ème }}$ siècles. 
Il nous a été possible de réunir trente-neuf de ces méthodes dont la date soit connue, listées dans la Figure 7. Dans ce corpus dominent largement les sources françaises et allemandes; les autres pays sont peu documentés. Il pourrait toutefois s'agir d'un effet de source dû au fait que les écrits répertoriés ici, lorsqu'ils ne sont pas accessibles directement en ligne, ont été édités sous la forme de fac-similés, mais avant tout pour ceux concernant la France. Ce corpus permet néanmoins un découpage chronologique fin des techniques enseignées puisque nous disposons donc de plusieurs sources pour quasiment chaque décennie d'existence de la clarinette entre le début du XVIII ${ }^{\mathrm{ème}}$ siècle et la fin du XIX ${ }^{\text {ème }}$ siècle. Les deux écrits d'Eisel et de Majer constituent des exceptions : ce ne sont pas à proprement parler des méthodes mais des ouvrages théoriques et encyclopédiques sur la musique. Ces sources présentent néanmoins pour nous l'intérêt, dans leur article consacré à la clarinette, de fournir chacune une tablature de jeu de la clarinette baroque : il s'agit des plus anciennes indications connues de jeu de la clarinette (Rice 1992).

Les premières pages de ces méthodes furent réellement pensées comme des supports pédagogiques, soit venant en complément de l'enseignement oral du professeur, soit tentant de se substituer au professeur dans le cadre d'une pratique amateur. Elles fournissent des descriptions de la posture à adopter faisant appel au vocabulaire de la mesure, sous la forme de textes d'une demi-page à une page, parfois accompagnés de dessins didactiques. Les tablatures indiquent en particulier comment placer les doigts sur l'instrument pour produire les différentes notes ; le plus souvent, un dessin de la clarinette l'accompagne (Figures 8 et 9). Ce dessin est didactique et non pas simplement illustratif, car on peut y trouver l'indication de la position des doigts sur les trous et les différentes clés, et du type de clarinette qui correspond aux doigtés indiqués. En effet, différents systèmes de clétage ont existé simultanément, et l'adjonction de clés est susceptible de modifier les doigtés à apprendre. Ainsi, on peut trouver les tablatures qui s'appliquent aux clarinettes à 6 et à 13 clés dans la même méthode (Carnaud 6). Le corpus comporte aussi des méthodes consacrées à d'autres instruments, comme la flûte traversière (Hotteterre 38), mais qui sont « augmentées »d'une tablature de clarinette, voire également de hautbois. 


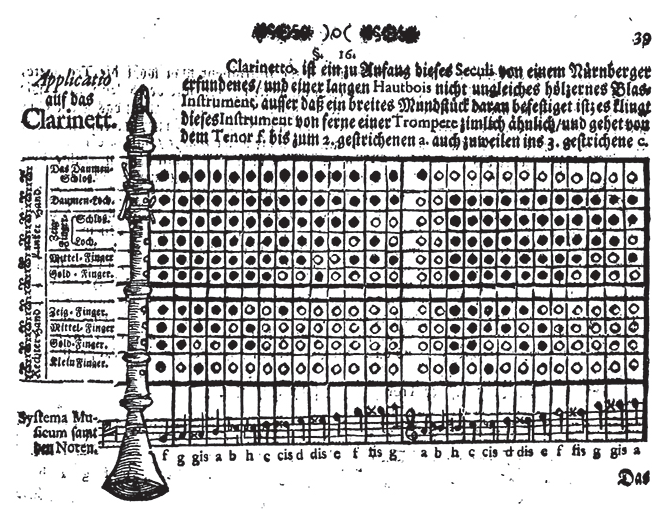

Figure 8 : Tablature de clarinette baroque. Joseph Majer, Museum Musicum Theoretico Practicum (1732), p. 39.

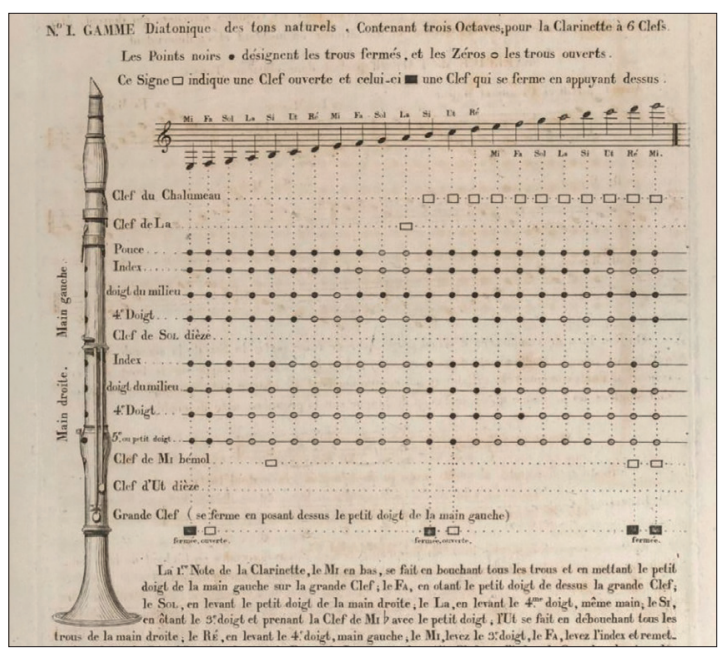

Figure 9: Tablature de clarinette classique. Jean Carnaud, Nouvelle méthode de la Clarinette moderne à 6 clefs et à 13 clefs (1829), p. 5.

\section{Reconstituer les gestes}

Le lien à double sens entre, d'une part, étude des sources afin d'identifier les gestes disparus, et d'autre part recréation de ces derniers (l'ensemble permettant une « histoire appliquée » à proprement parler), peut être explicité à travers le cas de la technique de Nicola Matteis. Ce violoniste napolitain est en effet venu s'installer à Londres à la fin du XVII ${ }^{\text {ème }}$ siècle pour profiter de l'absence de soliste de son envergure dans la capitale anglaise à cette époque. On sait par le témoignage de Roger North, amateur de musique ayant pris des notes sur les concerts qu'il a pu écouter, que sa technique a surpris les Londoniens : il tenait son violon « presque contre sa ceinture » (North 1715 76v) et « contre ses côtes courtes, [...] très loin de la manière habituelle de manipuler $»^{5}$ (North $172878 \mathrm{r}$ ). Quelques essais

« almost ag ${ }^{\mathrm{t}}$ his girdle »; « $\mathrm{ag}^{\mathrm{t}}$ his short ribbs, [...] much out of the comon road of handling ». Traduction personnelle. La question de l'identification anatomique, et partant, de la traduction exacte de «short ribbs », étant au cœur de la problématique sur ce cas, nous ne donnons à ce point de l'exposé qu'une traduction littérale. 


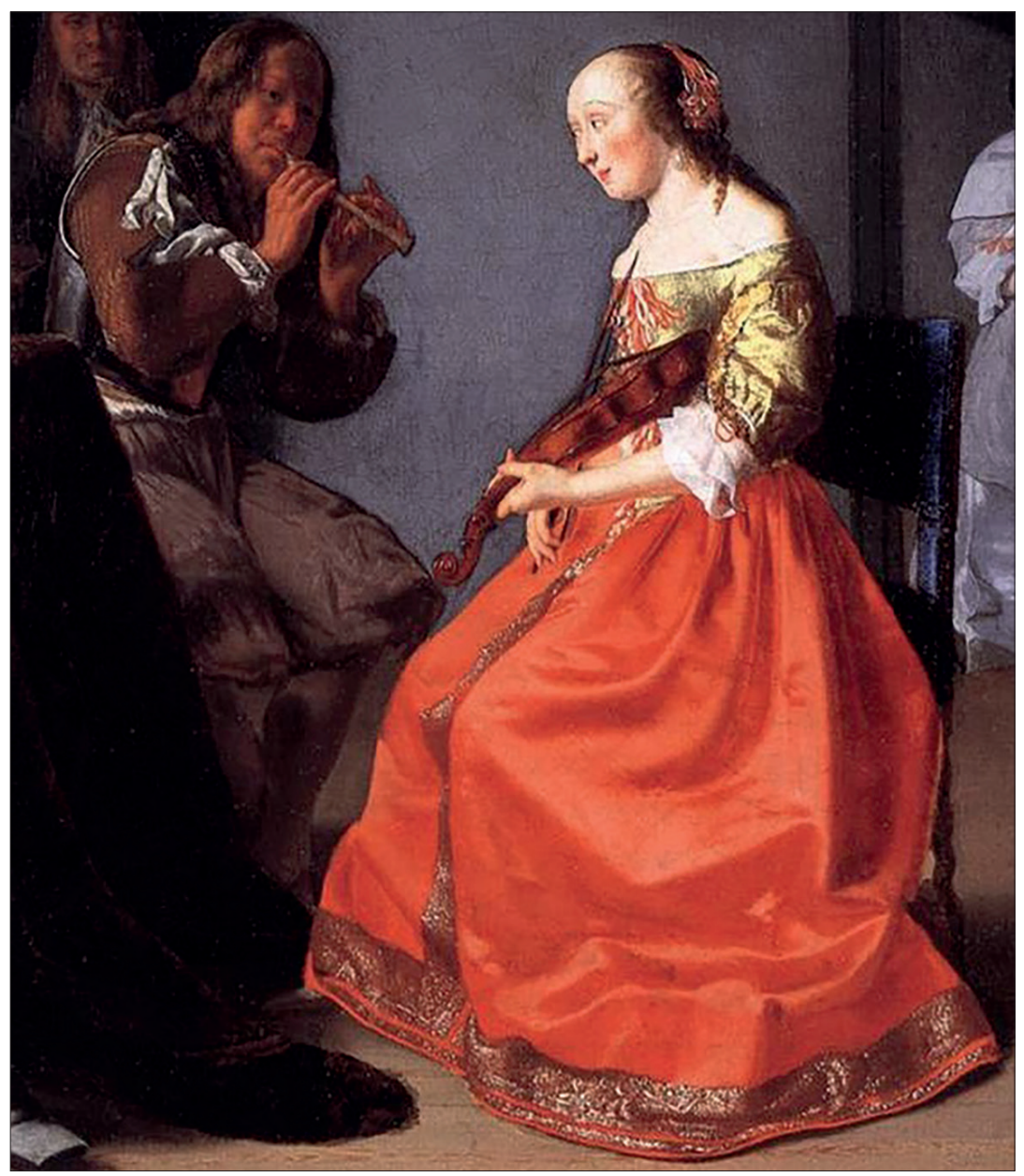

Figure 10: Jacob Ochtervelt, Compagnie musicale en intérieur (v. 1670). Huile sur toile, 59 x $49 \mathrm{~cm}$. Museum of Art, Cleveland. Détail. 
de restitution de cette technique ont été entrepris, notamment par Amandine Beyer (Beyer 2009), mais avec une analyse insuffisante du contexte historique : en effet, celle-ci assimile à "short ribbs " la clavicule (en occultant le pluriel de l'original) et tient donc son violon contre celle-ci, ce qui est effectivement une tenue basse et très inhabituelle par rapport aux standards actuels. Or, l'iconographie comme les sources textuelles anglaises de la fin du XVII ${ }^{\text {ème }}$ siècle, comme presque partout ailleurs en Europe d'ailleurs, décrivent explicitement le violon habituellement tenu contre l'épaule ou la poitrine. Matteis tenant son violon très bas (puisque vers sa ceinture) et non très haut, l'hypothèse de la clavicule est donc totalement impossible par rapport au contexte historique. En réalité, on peut identifier via les livres de chirurgie de l'époque que les « short ribbs » correspondent aux côtes flottantes, les plus basses de la cage thoracique (Van den Spiegel 108). Ceci correspond bien à la mention de la ceinture, une tenue extrêmement basse, et quelques peintures de figures féminines montrent l'instrument tenu sous la poitrine, soit de fait sur les côtes flottantes (Figure 10).

La reconstitution théorique de cette tenue suppose tout de même une validation expérimentale : en effet, Matteis était un soliste jouant de sa virtuosité et les œuvres qu'il a laissées emploient effectivement une technique complexe nécessitant des démanchés (cf : note 3 ) parfois importants. Or, comme le mentionne Léopold Mozart (cf : supra), une tenue basse est supposée rendre ceux-ci plus difficiles, et les interprètes actuels ont même tendance à considérer cette combinaison comme quasiment impossible. En effet, lorsque le menton ne tient pas l'instrument, la main gauche fait office de support et doit serrer le violon pour le presser contre le corps de l'instrumentiste, ne pouvant donc relâcher le manche pour reculer (avancer pose moins de difficultés). Là encore, le geste doit en réalité être interrogé, par exemple en rapport avec l'iconographie : alors que la technique actuelle interdit formellement de placer la main gauche dans une position horizontale, contraignant le musicien à adopter une tenue verticale, ce n'est pas le cas dans les représentations anciennes. Cette même main est ainsi très souvent représentée très aplatie, à tel point que l'intérieur du poignet peut venir toucher la caisse du violon et offrir un point d'appui, lequel autorise parfaitement le démanché avec une tenue très basse (celle-ci favorisant d'ailleurs cette position de la main, le bras étant trop court pour la placer verticalement).

Un équivalent plus subtil mais tout aussi crucial pour la clarinette est celui de la technique d'embouchure : celle qui est enseignée au sein des conservatoires est désormais fixée, mais l'analyse des sources permet de redécouvrir d'autres techniques. Au-delà de l'adaptation des doigtés aux systèmes de clétage antérieurs, jouer de la clarinette de manière historiquement informée nécessite de prendre en compte les techniques d'emploi du bec et de l'anche également, pour une approche de restitution historique plus rigoureuse et donc plus plausible pour l'interprétation. Outre le souci méthodologique, la facture de l'instrument est susceptible d'avoir une influence non négligeable sur les possibilités techniques de celui-ci et les habitus corporels qu'acquiert le musicien. Toutefois, ce n'est pas le seul 
paramètre en jeu. Les professeurs de clarinette et concertistes, en tant que figures de référence et d'autorité quant à ce qui constitue une belle performance à la clarinette, jouèrent également un rôle déterminant dans le processus de normalisation de la technique, en faisant école via l'enseignement oral au conservatoire et la rédaction des méthodes.

Si les différents auteurs intéressés par cette question se sont focalisés sur la position de l'anche (Hoeprich 1984 ; Rice 1992 ; Charlton 396) - soit vers le haut, c'est-à-dire tournée vers la lèvre supérieure, soit vers le bas, tournée vers la lèvre inférieure -, c'est la position et le rôle des lèvres et des dents qui sont déterminants. L'embouchure contemporaine consiste à tendre la lèvre inférieure et replier celle-ci sur les dents de la mâchoire inférieure, poser l'anche fixée au bec dessus, poser les dents de la mâchoire supérieure sur le bec et envelopper celui-ci avec les lèvres de façon à ce que l'air soufflé ne s'échappe pas par les côtés mais aille bien dans l'instrument. La technique dominante jusque dans les années 1850 et donc pratiquée sur les clarinettes à moins de treize clés (par opposition aux clarinettes à treize clés et plus par la suite) consiste à tenir le bec et l'anche entre les deux lèvres tendues uniquement, sans employer les dents. Dans cette configuration très différente, le clarinettiste pourrait dans l'absolu choisir le positionnement de l'anche selon sa préférence personnelle. Les sources fournissant des explications détaillées et des dessins sur l'embouchure montrent cependant que l'anche se tournait vers le haut (Fröhlich 12 ; Lefèvre planche 2 ; Van Der Hagen 1785 47) (Figure 11).

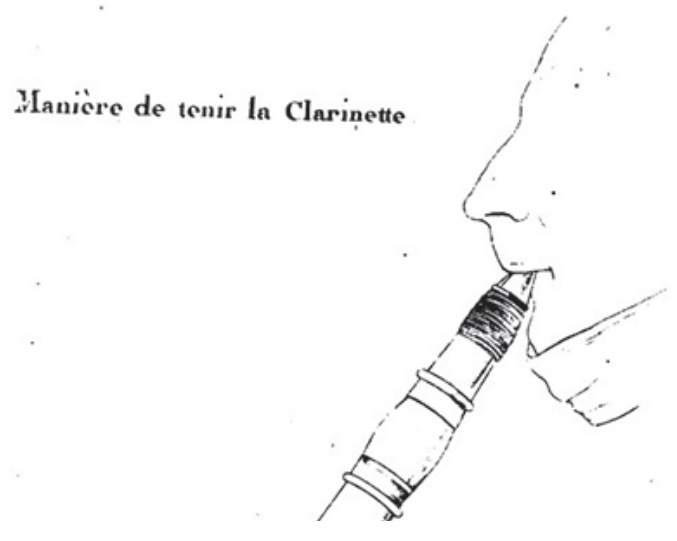

Figure 11 : Technique d'embouchure de clarinette classique. Jean-Xavier Lefèvre, Méthode de clarinette (1802), s. p. 
De plus, un savoir-faire, non musical à proprement parler, mais que les clarinettistes du XVIII ${ }^{2}{ }^{2}$ et du début du XIX ${ }^{\text {ème }}$ siècles devaient maîtriser, était la fabrication personnelle des anches. Si les méthodes insistent sur l'importance du choix du roseau et du soin apporté à la taille des anches, elles n'utilisent pas de vocabulaire précis, et l'évaluation de ce que sont un bon roseau et une bonne anche est très empirique. Les auteurs se contentent globalement d'indiquer de choisir du roseau « ni trop vert ni trop sec » (Berr 5) et de « se faire une anche douce pour commencer » (Van Der Hagen 47), mais peu fournissent des indications sur la manière de les faire, à trois exceptions près : Frédéric Berr, Jean-Xavier Lefèvre et François Rybicki. En 1826, Pierre Vaillant écrit même trois lignes dans sa méthode pour expliquer pourquoi il ne pose pas par écrit les étapes de la fabrication d'une anche. La raison qu'il donne est probablement commune aux autres auteurs : il estime que le support écrit ne convient pas et que l'apprentissage doit nécessairement passer par l'observation du professeur et le mimétisme jusqu'à arriver au résultat désiré. Ce cas montre les limites de l'utilisation des sources textuelles dans la restitution de pratiques musicales révolues. L'expérimentation est ici nécessaire pour pouvoir restituer une chaîne opératoire et se rapprocher des sons et des styles d'interprétation passés que l'on cherche à reproduire.

Par ailleurs, si les clarinettistes intéressés par la pratique de la clarinette historique adaptent donc leurs doigtés aux clarinettes de différentes factures, ils ne poussent pas la logique jusqu'au bout et continuent d'utiliser la technique d'embouchure contemporaine qu'ils ont apprise (parfois après avoir brièvement expérimenté l'embouchure ancienne ${ }^{6}$ ) ainsi que des anches standardisées produites industriellement. Au moment de la rédaction du présent article, nous n'avons en effet pas pu trouver d'interprétation utilisant la technique d'embouchure historiquement correcte. Un futur travail expérimental permettra donc d'évaluer l'importance de cette contradiction et de mettre en lumière dans quelle mesure une approche réellement rigoureuse est susceptible, en plus de modifier le rapport du corps à l'instrument, d'influer sur le rendu sonore des interprétations, à l'instar des recherches effectuées sur le violon.

On peut donc, à travers ces exemples, constater la nécessité d'effectuer des allers-retours entre l'analyse historienne des sources et l'expérimentation concrète des gestes identifiés, les deux réflexions se nourrissant mutuellement, et leur synthèse étant nécessaire pour aboutir à un résultat assuré et effectivement praticable dans le jeu musical. S'ils ne sont pas poussés jusqu'au bout, les différents essais de restitutions 《 archéologiques ${ }^{7}$ de situations musicales donnent pourtant un résultat généralement

Communication par e-mail d'Albert Rice, 2016.

Nous considérons ce terme comme impropre, mais il est habituellement employé par les spécialistes de musique ancienne pour désigner une restitution poussant la logique historique aussi loin que possible. 
beaucoup plus intéressant sur le plan artistique, et enthousiasmant pour le public, que les performances modernisées. Les représentations du Tartuffe inconnu en 2017, chantier de recherche autour d'une restitution du Tartuffe original de Molière ${ }^{8}$, peuvent en témoigner : d'après ses réactions pendant la représentation et a posteriori, le public, qui comprend instinctivement qu'il a le droit de parler pendant la musique (ce qui était normal à l'époque mais est interdit par les conventions actuelles), ressent clairement le dynamisme induit par l'exécution de musiques à danser avec la tenue basse du violon.

\section{Ouvrages Cités}

ABRAHAM. Principes de clarinette. Paris : Frère, c. 1782. [Reproduction en fac-similé]. Courlay : Fuzeau, 2000. 43-44.

ANONYME. Apollo's Cabinet: or the Muse's delight. An accurate collection of english and italian songs, cantats and duetts, set to music for the Harpsichord, Violin, German-Flute, \&c. With instructions for the Voice, Violin, Harpsichord or Spinet, German-Flute, Common-Flute, Hautboy, French-Horn, Basson, and Bass-Violin. Also, a compleat musical dictionary, and several hundred english, irish and scots songs, without the music. Liverpool : John Sadler, 1756.

ANONYME. Gamme de la clarinette. Paris : s.n., 1790. [Reproduction en fac-similé]. Courlay : Fuzeau, 2000. $67-70$.

ANONYME. The Clarinet Instructor by Which Playing on That Instrument Is Rendered Easy to anyone unacquainted with Music as it contains a Compleat Scale. Capital Performer on the above Instrument, to which is added Six new Duos for two Clarinets and a quintetto for Horns, Clarinets and a Bassoon. Londres : Longman \& Broderip, 1780.

ADLUNG, Jacob et Johann Ernst BACH. Anleitung zu der Musikalischen Gelehrtheit. Erfurt : J. D. Jungnicol, 1758.

ALBRECHTSBERGER, Johann Georg. Gründliche Anweisung zur Composition...mit einem Anhange : von der Beschaffenheit und Anwendung aller jetzt üblichen Musikalischen Instrumente. Leipzig : Johann Gottlob Emmanuel Breitkopf, 1790.

ANTOLINI, Francesco. La retta maniera di scrivere per il clarinetto ed altri istromenti da fiato, Milan : C. Buccinelli, 1813.

Cette restitution concernait aussi bien la pièce en elle-même que sa prononciation, sa mise en scène, le jeu des acteurs, mais également les entractes égayés par une bande de violons jouant des musiques à la mode au moment de sa représentation, avec un effectif orchestral et une technique de jeu historiquement documentés. 
ARBEAU, Thoinot. Orchésographie, metode, et teorie en forme de discours et tablature pour apprendre à dancer, battre le tambour en tout sorte \& diversité de batteries, jouër du fifre \& arigoy, tirer des armes \& escrimer, avec autres honnestes exercices fort convenables à la jeunesse. Affin d'estre bien venue en toute joyeuse compagnie \& y monstrer sa dexteritè \& agilité de corps. 1589. Lengres: Iehan des Preyz, 1596.

B., T. The compleat Musick-Master: being Plain, Easie, and Familiar Rules for Singing, and Playing on the most useful Instruments now in Vogue, according to the Rudiments of Musick. Viz., Violin, Flute, Haut-Boy, Bass-Viol, Treble-Viol, Tenor-Viol. Containing likewise A great Variety of Choice Tunes, and fitted to each Instrument, with Songs for two Voices. To which is added, a Scale of the Seven Keys of Musick, shewing how to Transpose any Tune from one Key to another. Londres : William Pearson, 1722.

BACKOFEN, J. G. H. Anweisung zur Clarinette mit besonderer Hinsicht auf die in neuern Zeiten diesem Instrument beigefügten Klappen nebst einer kurzen Abhandlung über das Basset Horn. Breitkopf et Härtel : Leipzig, 1824.

BAERMANN, Carl. Vollständige Clarinett Schule von dem ersten Anfang bis zur höchsten Ausbildung des Virtuosen. Offenbach : J. André, 1865-75. 2 vols.

BEER, Frédéric. Méthode complète de clarinette. Paris : Désiré Kelmer, 1891.

—. Traité complet de la clarinette à quatorze clefs. Paris : E. Duverger, 1836.

—. Méthode complète de clarinette à quatorze clefs. Paris : J. Meissonnier, 1836.

BEYER, Amandine. Matteis: False Consonances of Melancholy. Paris : Zig-zag Territoires, 2009.

BLAKE, G. E. A new and complete preceptor for the clarinet, Being an Introduction to the Art of Playing on that Instrument Explained in the most simple and comprehensive manner and A selection of the newest \& most Favorite Airs, Song tunes, Marches. Philadelphia : s. n., 1804.

BLASIUS, Frédéric. Nouvelle méthode de clarinette et raisonnement des instruments. Paris : Porthaux, 1796. [Reproduction en fac-similé]. Courlay : Fuzeau, 2000. 81-196.

BLAZICH, Joan. Amand Vanderhagen's Méthode nouvelle et raisonnée pour la clarinette (1785) and Nouvelle méthode de clarinette (1796): Complete Translations and Analyses of the First Classical Clarinet Treatises. Thèse de doctorat en musicologie. Cincinatti : Université de Cincinatti, 2005.

BOCHSA, Charles. Méthode instructive pour la clarinette. Paris : Imbault, 1808.

BOSCHER, A. Méthode de $2^{e}$ clarinette en si bémol (extraite de la méthode générale d'ensemble). Paris : David, 1875.

—. Méthode de petite clarinette en mi bémol (extraite de la méthode générale d'ensemble). Paris : David, 1875.

BOUILLON, Paul. Méthode élémentaire pour la clarinette. Paris : Union musicale, 1876. 
BOYDEN, David. The History of Violin Playing From its Origins to 1761 and its Relationship to the Violin and Violin Music. 1965. Oxford : Oxford University Press, 1990.

CARNAUD. Nouvelle méthode de la clarinette moderne à six et à treize clefs. Paris : Collinet, 1829.

CHARLTON, David. «Classical Clarinet Technique: Documentary Approaches ». Early Music 16 (1988) : 396-406.

CORNETTE, Victor. Méthode de clarinette. Paris : Colombier, 1855.

CORRETTE, Michel. L'École d'Orphée. Méthode pour Apprendre facilement à joüer du Violon Dans le goût François et Italien; Avec des Principes de Musique Et beaucoup de Leçons à I, et II Violons. Ouvrage utile aux commençants et a ceux qui veulent parvenir à l'execution des Sonates, Concerto, Pieces par accords Et Pieces a cordes Ravallées. Paris: L'Auteur, Boivin et Le Clerc, 1738.

- Méthode raisonnée pour apprendre aisément à jouër de la Flûtte traversiere avec les principes de Musique, des Aroettes et autres Jolis Airs en Duo. Ouvrage utile et Curieux qui conduit en très peu de tems a la parfaite connoißansse de la Musique et à Jouer à Livre ouvert les Sonates, Concerto, et Symphonies. Nouvelle édition, revûe, corrigée et augmentée de la Game du Haut-bois et de la Clarinette. s. 1. : s. n., 1773.

- L'Art de se perfectionner dans le Violon. Ou l'on donne à étudier des Leçons sur toutes les positions des quatre cordes du Violon et les différens coups d'archet. Ces leçons où les doigts sont marqués dans les endroits difficiles, sont tirées des Sonates et Concerto des meilleurs Auteurs Italiens et allemands \&c. avec des préludes sur Chaque ton, des points d'orgues, des tours de forces, des Menuets et Caprices avec des Variations et la Basse. Cet Ouvrage faut la Suite de l'Ecole d'Orphée Méthode pour le Violon. Paris: $\mathrm{M}^{\text {elle }}$ Castagnery, 1782.

CROME, Robert. The Fiddle New Model'd or a useful Introduction to the Violin, Exemplify'd with familiar Dialogues. Londres : J. Thyler, 1735.

DE BROSSARD, Sébastien. [Fragments d'une méthode de violon]. s.1. : v. 1711. [Reproduction en fac-similé]. Courlay : Fuzeau, 2003. 23-54.

DE HERRANDO, Joseph. Arte y puntual explicación del modo de tocar el violín. Para qualquiera que aprenda asi aficionado como Professor aprovechándos los maestros en la enseñanza de fus discípulos, con más brevedad y descanso. Paris : Joannes a Cruce, 1756.

DE LABORDE, Jean-Benjamin. Essai sur la musique ancienne et moderne. Tome premier. Paris : Ph.-D. Pierres, 1780. [Reproduction en fac-similé]. Courlay : Fuzeau, 2003. 237-240.

DÉMAR, Sébastian. Nouvelle méthode pour la clarinette. Orléans : Démar Sébastian, 1808.

DUBOURG-GLATIGNY, Pascal et Hélène VÉRIN (dirs.). Réduire en art. La technologie de la Renaissance aux Lumières. Paris : Maison des Sciences de l'Homme, 2008. 
EISEL, Johann Philipp. Musicus Autodidactos oder der sich selbst Informierende Musicus, Erfurt : Johann Michael Funcken, 1738.

FALCK, Georg. I. N. SS. T. Idea Boni Cantoris, das ist: Getreu und Gründliche Anleitung, Wie ein MusicScholar, so wol im Singen, als auch auf andern Instrumentis Musicalibus in kurtzer Zeit so weit gebracht werden kan, daß er ein Stück mit-zusingen oder zu spielen sich wird unterfangen dörffen; Aus Verschiedenen berühmten Musicis colligirt, und der Music-Liebenden Jugend zu sonderbahrer Lust-Erweck-und nutzlichen Begreiffung zusammen geschrieben, und heraus gegeben. Nürnberg : Wolffgang Moris Endter, 1688.

FRÖHLICH, Joseph. Vollständige theoretisch-praktische Musikschule. Bonn : s.n., 1810.

GAMBARO, Jean-Baptiste. Méthode de clarinette à six clefs avec la manière de faire les anches. Paris : Gambaro, 1840.

GEMINIANI, Francesco. The Art of Playing on the Violin. Containing All the Rules necessary to attain to a Perfection on that Instrument, with great variety of Compositions, which will also be very useful to those who study the Violoncello, Harpsichord \&c. Londres : John Johnson, 1751.

GÉTREAU, Florence. Voir la musique : Les sujets musicaux dans les œuvres d'art du XVI ème au XX'̀me siècle. Musée départemental de l'Abbaye de Saint-Riquier, Musée de Millau : Musée des Beaux-Arts de Carcassonne, 2009.

HOEPRICH, Eric. "Clarinet Reed Position in the 18th Century ». Early Music 12:1 (février 1984) : 48-55.

—. « The Earliest Paintings of the Clarinet ». Early Music 23:2 (mai 1995) : 258-266.

HOTTETERRE, Jacques. Méthode pour apprendre à jouer en très peu de tems de la Flute traversière, de la Flute à bec et du Haut-bois... Augmentée des Principes de la musique et des Tablatures de la Clarinette et $d u$ Basson. Paris : Bailleux, 1775. [Reproduction en fac-similé]. Courlay : Fuzeau, 2000. 38.

HOWE, Elias. Howe's New Clarionet Instructor. Boston : Oliver Ditson \& Company, 1850.

JAMBE DE FER, Philibert. Epitomé musical des tons, sons et accordz, ès Voix humaines, Fleustes d'Alleman, Fleustes à neuf trous, Violes, \& Violons. Item. Vn petit deuis des accordz de Musique; par forme de dialogue interrogatoire \& responsif entre deux interlocuteurs. P. \& I. Lyon : Michel du Bois, 1556.

KELLNER, Franz. Méthode complète pour la clarinette Boehm et ordinaire. Paris : Alphonse Leduc, 1868.

—. Méthode complète pour la clarinette Boehm et ordinaire, $2^{e}$ partie. Paris : Alphonse Leduc, 1879.

KÜRZINGER, Ignaz. Getreuer Unterricht zum Singen mit Manieren, und die Violin zu spielen. Zum Gebrauch und Nuzen der Anfänger ; zur Erleichterung der Herren Chorregenten, Cantorn, Thurnermeistern, und andern, die sich mit Instruieren beschäftigen. Nebst einem Alphabetischen Anhang der mehrsten Sachen, welche einem rechtschaffenen Sänger, oder Instrumentisten zu wissen nöthig sind. 1763. Augsburg: Johann Jacob Lotter und Sohn, 1793. 
LABADENS, Jean-Baptiste. Nouvelle Méthode pour apprendre à jouer du Violon, et à lire la Musique ; enrichie de plusieurs Estampes en taille douce. Paris : Adresses Ordinaires de Musique, 1772. [Reproduction en fac-similé]. Courlay : Fuzeau, 2003. 13-84.

L'ABBÉ LE FILS (SAINT-SEVIN, Joseph-Barnabé). Principes du Violon pour apprendre le Doigté de cet Instrument, et les différends Agréments dont il est susceptible. 1761. Paris : Des Lauriers, 1772. [Reproduction en fac-similé]. Courlay : Fuzeau, 2003. 85-170.

LACHEZE, Cyril. La Tenue du violon à l'époque baroque. Université Paris 1 : Mémoire de Master II d'Histoire, 2013. 2 vols.

LAWSON, Colin (ed.). The Cambridge Companion to the Clarinet. Cambridge : Cambridge University Press, 2001.

LEFÈVRE, Jean-Xavier. Méthode de clarinette. Paris : Naderman, 1802.

LENTON, John. The Gentlemen's Diversion, or The Violin Explained. Londres : Lenton, 1693.

LÖHLEIN, Georg. Anweisung zum Violinspielen, mit praktischen Beispielen und zur Uebung mit vier une zwanzig kleinen Duetten erläutert. 1774. Leipzig und Züllichau : Waysenhausund Frommannischen Buchhandlung, 1781.

MAGNANI, Aurelio. Méthode complète de clarinette système Boehm. Paris : Evette et Schaeffer, 1900. 3 vols.

MAJER, Joseph. Museum Musicum Theoretico Practicum, das ist, Neu-eröffneter Theoretischund Practischer Music-Saal, darinnen gelehret wird wie man sowohl die Vocalals Instrumental-Music gründlich erlernen, auch die heut zu Tag üblichund gewöhnlichste, blasend, schlagend und streichende Instrumenten in kurzer Zeit und compendieuser Application in besondern Tabellen mit leichter Mühe begreifen könne. Nebst einem Appendice derer anjeßo gebräuchlichst Griechisch Lateinisch Italiänisch und Französisch musicalischen Kunst-Wörter nach Alphabetischer Ordnung eingerichtet und erkläret. Zum nutzlichen Gebrauch aller und jeder Music-Liebhaber zusammen betragen und mitgetheilet. Schwäbisch-Hall : Georg Michael Majer, 1732.

MERCK, Daniel. Cempendium Musica Instrumentalis Chelica. Das ist : Kurtzer Begriff, Welcher Gestalten Die Instrumental-Music auf der Violin, Pratschen, Viola da Gamba, und Bass, gründlich und leicht zu erlernen seye. Der Jugend und andern Liebhabern zu Gefallen aufgesetzt, und auf Begehren guter Freunde zu offentlichem Druck befördert. Augsburg : Johann Christoph Wagner, 1695.

MOENS-HAENEN, Greta. Deutsche Violintechnik im 17. Jahrhundert. Ein Handbuch zur Aufführungspraxis. Graz : Akademische Druck- und Verlagsanstalt, 2006.

MOZART, Léopold. Méthode raisonnée pour apprendre à Jouer du Violon. Paris : Le Menu, 1770. [Reproduction en fac-similé]. Courlay : Fuzeau, 2003. 231-323. 
-.Versuch einer gründlichen Violinschule, entworfen und mit 4. Kupfertafeln sammt einer Tabelle versehen. Augsburg : Johann Jacob Lotter, 1756.

MUFFAT, Georg. Suavoris Harmoniae Instrumentalis Hyporchematicae Florilegium Secundum. Passau : Georg Adam Höller, 1698.

MÜLLER, Iwan. Anweisung zur der neuen Clarinette und der Clarinette-alto, nebst einigen Bemerkungen für Instrumentenmacher. Leipzig : Friedrich Hofmeister, 1821.

NADAUD, L. Méthode pour la clarinette. Paris : Nadaud, 1841.

—. Méthode pour la clarinette à 6 et 13 clefs. Paris : Joly, 1851.

NORTH, Roger. An Essay on Musicall Ayre. S.1. : s.n., 1715.

—. The Musicall Gramarian. S.1. : s.n., 1728.

PEARSON, Ingrid E. « $18^{\text {th }}$ - and $19^{\text {th }}$-Century Iconographical Representations of Clarinet Reed Position ». Music in Art 25:1/2 (printemps-automne 2000) : 87-96.

PIGNOLET DE MONTECLAIR, Michel. Méthode Facile pour apprendre à joüer du Violon avec un Abregé des Principes de Musique necessaires pour cet Instrument. Paris : L'Auteur, 1711. [Reproduction en facsimilé]. Courlay : Fuzeau, 2003. 55-68.

PLAYFORD, John. A Brief Introduction to the Skill of Musick. In two Books. The First containes the General Grounds and Rules of Musick. The Second, Instructions for the Viol and also for the Treble-Violin. Londres : William Goodbid, 1664.

PRAETORIUS, Michael. Syntagmatis Musici. Tomus Secundus. De Organographia. Wolfenbüttel : Elias Holwein, 1618.

PRINNER, Johann. Musicalischer Schlissl. Salzbourg : s.n., 1677.

RICE, Albert. The Baroque Clarinet. Oxford : Oxford University Press, 1992.

—. The Clarinet in the Classical Period. Oxford : Oxford University Press, 2003.

ROESER, Valentin. Principes de clarinette. Avec la Tablature des Meilleurs Maîtres pour cet Instrument et plusieur Duo pour cet Instrument. Paris : s.n., 1760. [Reproduction en fac-similé]. Courlay : Fuzeau, 2000. 5-11.

RYBICKI, François. Méthode pour la clarinette. Paris : Arnaud, 1826.

SPEER, Daniel. Grund-richtiger, kurtz, leicht und nöthiger Unterricht der Musikalischen Kunst, Wie man füglich und in kurtzer Zeit Choral und Figural singen, den General-Baß tractiren, und Componiren lernen soll. Ulm : Wilhelm Kühne, 1687. 
TARADE, Théodore-Jean. Traité du Violon ou Regles de cet Instrument A l'usage de ceux qui veulent en jouer avec la parfaite connoissance du Ton dans lequel on est. Paris : Girard, 1774. [Reproduction en fac-similé]. Courlay : Fuzeau, 2003. 171-236.

TRICHET, Pierre. Traité des instruments de musique. s.1. : s.n., 1640.

VAILLANT, Pierre. Nouvelle méthode de clarinette à cinq et à treize clefs. Paris : Pierre Vaillant, 1826.

VAN DEN SPIEGEL, Adrian. The Workes of that Famous Chrurgion Ambrose Parey. Londres : Richard Cotes et Willi Dugard, 1649.

VAN DER HAGEN, Amand. Méthode nouvelle et raisonnée pour la clarinette. Paris : Boyer, 1785. [Reproduction en fac-similé]. Courlay : Fuzeau, 2000. 45-64.

—. Nouvelle méthode de clarinette divisée en deux parties. Paris : Pleyel, 1796. [Reproduction en fac-similé]. Courlay : Fuzeau, 2000. 197-274.

VILLE, Paul, de (dir.). Lazarus Clarinet School: A New and Modern Method for Clarinet Boehm and Ordinary System, Revised, Compiled and Enlarged. Londres : Carl Fischer, 1881, 3 vols.

WESTON, Pamela. Clarinet Virtuosi of the Past. Londres : R. Hale, 1971.

—. More Clarinet Virtuosi of the Past. Londres : Weston Pamela, 1977.

YOST, Michel. Méthode de clarinette. Paris : s.n., 1800. [Reproduction en fac-similé]. Courlay : Fuzeau, 2000. 275-300.

Résumé : Nous nous proposons à travers cette communication d'analyser les rapports entre les gestes musicaux dits anciens, autrement dit oubliés dans la pratique actuelle et redécouverts par une étude de type historique, et les sources de natures textuelle et iconographique utilisées pour cela. Nous interrogeons cette interaction de manière bidirectionnelle, à savoir les apports des sources pour la compréhension des gestes dans la recherche actuelle, mais également les modalités d'inscription du geste dans les œuvres au moment de leur création. La première partie de notre intervention porte sur la période moderne à travers l'exemple du violon. Les sources textuelles et iconographiques sont dans ce cas disjointes : textes didactiques d'une part, peu ou pas illustrés, et œuvres picturales artistiques d'autre part. Dans ce cas, les modalités de transcription du geste dans la source sont différentes selon les œuvres, de même que les informations apportées, par conséquent plus ou moins complémentaires. Dans une seconde partie, nous abordons le XIX ${ }^{\text {ème }}$ siècle via le cas de la clarinette, instrument plus tardif dans l'histoire de la musique. Ici, il existe deux types d'écrits différents intégrant tous deux une iconographie. D'une part, les ouvrages didactiques sont maintenant illustrés, et d'autre part un certain nombre de brevets d'invention, prenant également le geste en compte, présentent à la fois un texte et une image normatifs. Ainsi, cette étude permet de saisir sur un temps long, quatre siècles, les évolutions du rapport entre gestes et 
représentations, textuelles et iconographiques, dans le domaine de la technique musicale. Les représentations de la période moderne sont ainsi marquées par une prégnance importante de l'imaginaire de la technique. Au contraire, celles du XIX ${ }^{\mathrm{ème}}$ siècle, nettement plus ancrées dans le réel, sont devenues le vecteur de luttes d'intérêt de nature socio-technique. Elles développent également la notion d'une technique abordable non plus par l'écrit seul ou illustré, mais également par l'image uniquement : on peut trouver un écho de cette conception dans la pratique technique réelle de ces instruments, passant entre autres par le visuel et que nous nous proposons de faire dialoguer avec les représentations discutées.

\begin{abstract}
This article studies how gestures used in the past to play musical instruments can be reconstructed with the help of textual and visual sources. It examines on the one hand the way sources can be used to find out about the gestures made by musicians in the past, and on the other hand, how gestures were represented in art works and texts. The first part of the article deals with the violin in the early modern period. Researchers use separate sources, first didactic handbooks, without any illustrations, secondly art works representing violin players. In this case, the information retrieved from books can be supplemented by the art works. The second part deals with the nineteenth century and a later instrument, the clarinet. The case is different, as didactic manuals were now illustrated and the invention of the instrument left traces in patents that explained how to play the clarinet, via images and text. In this way, we are able to give a survey of relations between gestures, texts and images, over four centuries in the domain of musical interpretation. The earlier period emphasises technicity, whereas the later period is the locus of social as well as technical conflicts. The article also considers how musicians were likely to learn how to play by observing the gestures made by performers, and so focuses on actual practice alongside literary and artistic representation.
\end{abstract}

